\title{
Simulation of Semiconductor Manufacturing Equipment and Processes
}

\author{
Siqing Lu \\ Samsung Electronics Co., Ltd. \\ 1-1 Samsungjeonja-ro, Hwaseong-si, Gyeonggi-do, Korea 18848 \\ siqing.lu@samsung.com
}

\begin{abstract}
In this paper, an overview of thermal, flow and plasma simulations of semiconductor manufacturing equipment and processes is presented. Semiconductor devices have been the major driver of the IT revolution for decades and their already complicated manufacturing processes become more and more challenging as the critical dimension goes down. Specific pieces of equipment have to be developed for specific processes, most of which involves interactive heat transfer, gas dynamics and chemical reactions. Even though equipment developments still mainly rely on DOE (design of experiments), simulations play an increasingly significant role. This paper describes some key aspects and insights in this area.
\end{abstract}

Keywords: heat transfer, gas dynamics, plasma, CFD, simulation, semiconductor manufacturing equipment, nanotechnology

\section{Introduction}

Semiconductor devices such as CPU/AP, DRAM and SSD have enabled the IT revolution and continue to be a major driver of new technologies and industries. As the critical dimension of the semiconductor devices goes to sub-10nm, it becomes an extremely challenging task to understand the nano-scale gas transport and reaction phenomena within such small features. Moreover, macro-scale transport phenomena must also be studied cross the whole wafer (currently of $300 \mathrm{~mm}$ diameter) to achieve high yield of semiconductor production.

It must be admitted that the understanding of heat transfer and fluid flow of manufacturing processes of semiconductor devices has never been mature due to its complexity and fast changing characteristics of the industry. We still largely rely on trial-and-error and DOE (design of experiments) for process evaluation and tuning. This paper, from an engineer's point of view, describes the application of thermal, flow and plasma simulations that have significant impacts on product development [1].

\section{Process Chambers and Multi-Physics Simulations}

To manufacture a semiconductor device such as a CPU chip, hundreds of process steps are needed that employ dozens of processing equipment for lithography, diffusion, implantation, etch, deposition, metallization, chemical-mechanical polishing, clean and etc. While high temperature $\left(600-1200^{\circ} \mathrm{C}\right)$ thermal processes may be used to meet the energy level requirements and to achieve fast growth rates in pre-metal steps, the processing temperature must be limited after the first interconnect lines (currently copper) are deposited to avoid melting the metal. In this case, RF (radio frequency), MW (microwave), or other power can be used as additional or major energy source that energizes gases and generates plasma to obtain adequate reaction rates. Then mass transport and reaction mechanisms involve internally-coupled thermal, flow and plasma at multiple time and geometric scales.

Fig. 1 presents a schematic of polysilicon etch chamber, in which reactive and carrier gases are delivered through nozzles, a turbo pump is used to pull out the gas to maintain a vacuum chamber pressure about 1-10 Pascal, RF coils provides energy to generate ICP (inductively-coupled plasma), and ESC (electro-static chuck) with bias voltage connected to modulate the temperature of wafer and the directionality of ions.

Thermal simulation can produce temperature distribution of components and silicon wafer that are affected by conduction, convection, radiation and ion bombardment. Gas concentrations of different species on the wafer can be simulated by CFD (computerized fluid dynamics) software. Plasma ion densities of various species can be simulated by 
EM field and electron impact reactions. Finally, the evolving process of silicon etch with photo-mask is simulated using Mont-Carlo method using the boundary condition produced by plasma simulation [2].

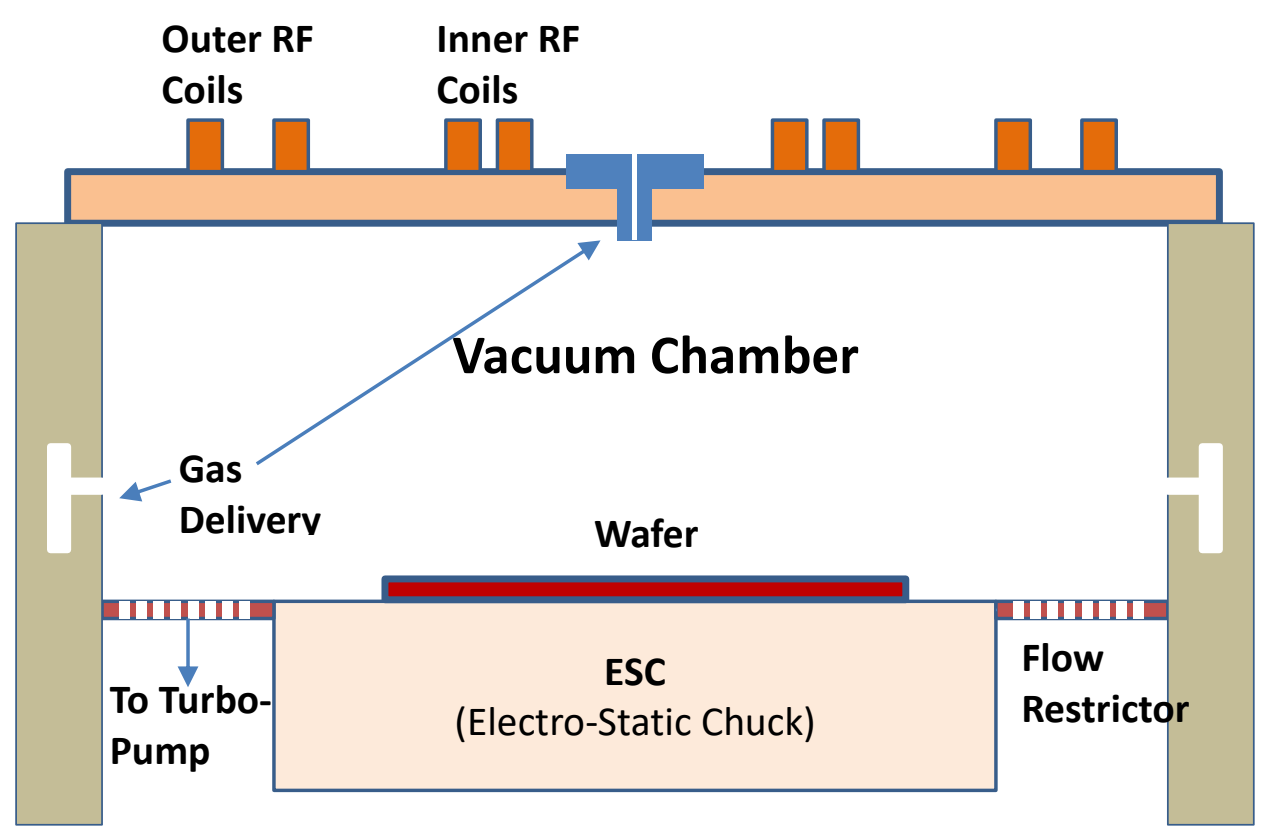

Fig. 1: Schematic of Poly Etch Chamber for Multi-Physics Simulation.

\subsection{Heat Transfer Simulation}

The temperature distribution of the silicon wafer is one of the most important parameter in manufacturing process of semiconductor devices. Differentiation of wafer temperature from other components is a commonly used to produce the desired deposition/etch rate on the wafer while minimizing by-product deposition/corrosion of other chamber components. The conductive heat transfer between wafer and its susceptor is normally dependent of gas pressure dependent because the mean-free path at chamber vacuum is at the same scale of the gap dimension. Therefore, modulation of wafer backside gas pressure may be necessary to provide sufficient heat transfer that couples the wafer with susceptor much stronger than with the surrounding components. Otherwise, wafer temperature may drift when the surrounding emissivity changes due to byproduct deposition/corrosion. Ion bombardment on solid surface can be estimated by ion densities and sheath voltage produced by plasma simulation. For RTP (rapid thermal processing) chambers, the degradation of radiation due to the coating on the transparent quartz dome can also be simulated by characterizing its transparency and emissivity.

\subsection{Gas Flow Simulation}

To meet the requirement of $1 \%$ film non-uniformity cross the whole $300 \mathrm{~mm}$ wafer, gas flow must be well controlled and modulated. Under low chamber pressure such as in etcher, the gas diffusions are great and thus the flow uniformity can be more readily achieved by using just a few nozzles. On the other hand, for high pressure processes such as SACVD (subatmosphere chemical vapor deposition) a showerhead with more than 10,000 holes has to be used. Especially when there is plasma, the gas species includes neutrals and ions and thus the flow uniformity can be affected by both transportation and electric field. To improve the uniformity further, the gases may be supplied separately from center and edge or through multiple zones. 


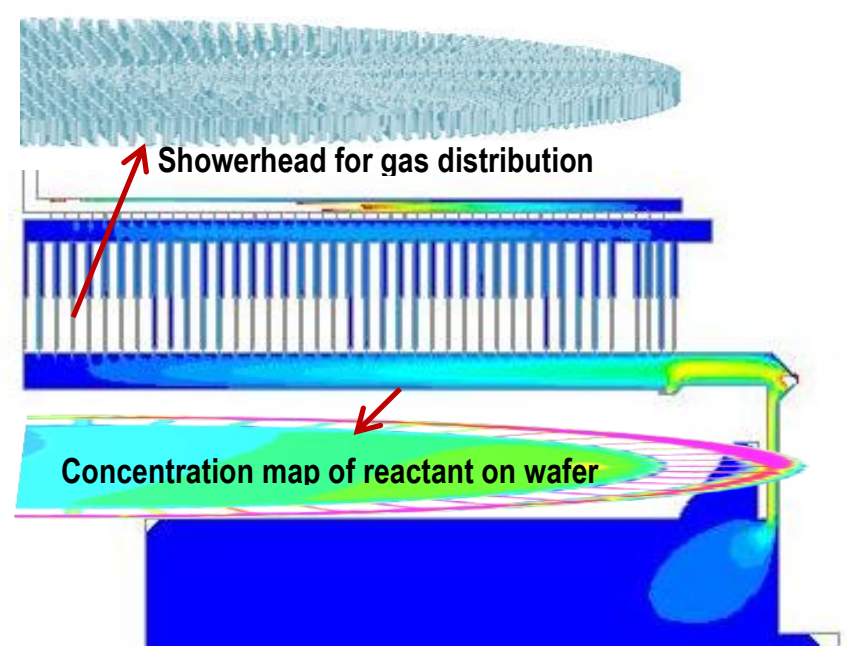

Fig. 2: Gas flow simulation of a SACVD chamber.

\subsection{Reaction Simulation}

Volumetric reactions produce necessary gas species and surface reactions on the wafer grow or remove the required film. There are many intermediate species/reaction steps for deposition and etch in semiconductor manufacturing process, especially when remote or in-situ plasma is generated. It is critical to simulate the all reactions for better understanding the deposition/etch mechanisms. Fig. 3 presents the reaction pathways of simple $\mathrm{NF}_{3} / \mathrm{O}_{2}$ mixture using remote plasma, where $\mathrm{M}$ is $3^{\text {rd }}$ body. It has been found from the simulation that FNO plays an important role for this silicon nitride dry clean process.

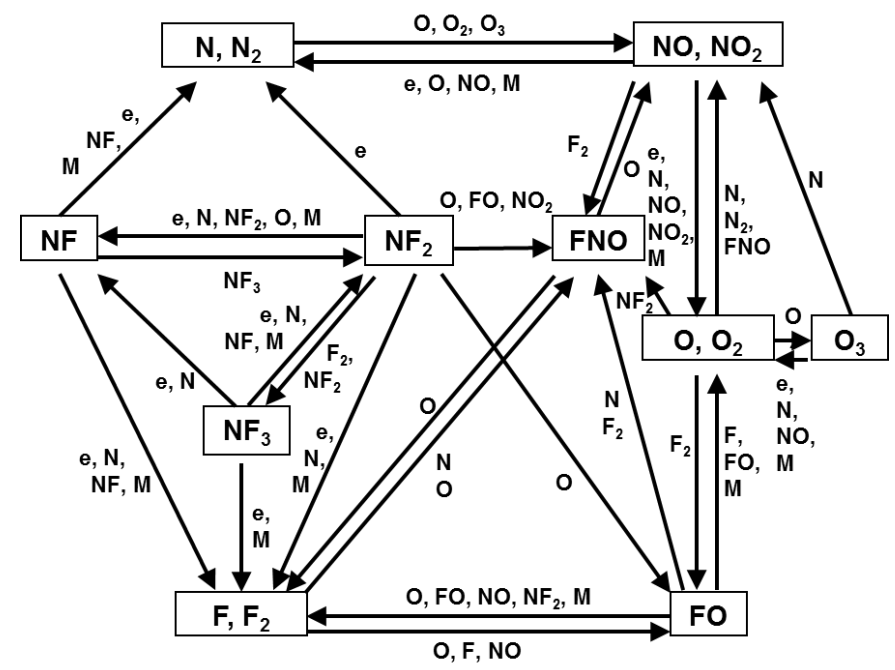

Fig. 3: Reaction mechanism with $\mathrm{NF}_{3} / \mathrm{O}_{2}$ remote plasma.

\subsection{Nano-Scale Profile Simulation}

As described earlier, the CD of semiconductor devices has now at about 10nm. Deposition and etch processes in a device feature such as a trench can also be simulated. With a chamber pressure at $1 \mathrm{~Pa}$ level and the characteristic length at nanometer level, the neutral transport is by molecular flow. The transport of gas molecules through trench top to bottom is dependent of side wall sticky coefficient. To simulate the etch process, gas phase species can be represented by Monte Carlo pseudo-particles, which are launched with energies and angles sampled from the distributions obtained from plasma simulation.

Fig. 4 shows a simplified example of $\mathrm{Cl}_{2}$ etch of $\mathrm{Si}$ to form a trench (with mask). It is a useful tool for understanding the RIE (reactive ion etch) processes. 


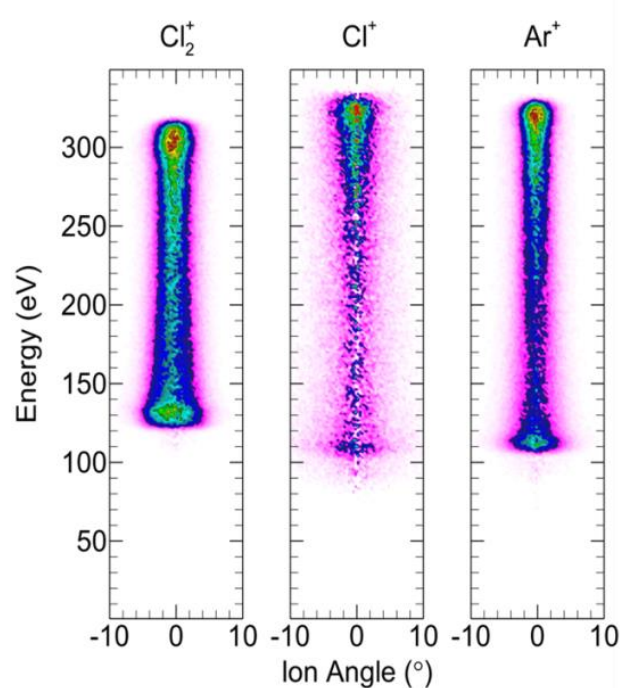

(a)

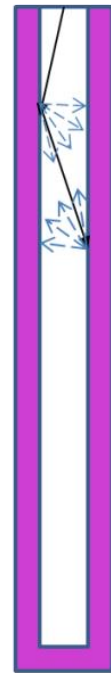

(b)

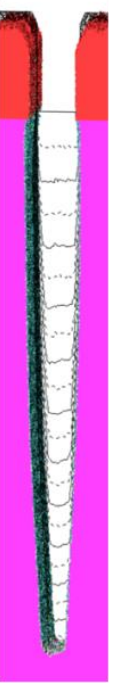

(c)

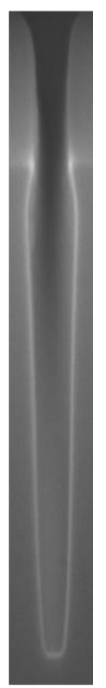

(d)

Fig. 4: (a) Ion energy distribution; (b) gas transport within the trench; (c) etch evolving process; (d) tested etch profile

Fig. 4: Simulation of reactive ion etching of $\mathrm{Si}$ using $\mathrm{Cl}_{2}$.

\section{Conclusion}

It is a very challenging task to simulate the semiconductor manufacturing processes that involves coupled multiphysics including heat transfer, continuous or rarefied gas dynamics, plasma, and chemical reactions. Currently there are no commercial software packages that can simulate all the physics simultaneously and de-couplings must be considered to simulate the processes by using single physics. This in turn may make the simulation results deviated from real world conditions.

There is a large knowledge gap between the properties of deposited/etched films and controllable parameters of the process equipment. Simulations are often used to reveal the unknown intermediate process parameters, build up the corrections, and thus bridge the knowledge gaps. It is no surprise that the quality of simulations largely depends on simulation engineer's understanding of the underlying sciences.

\section{Acknowledgements}

I greatly appreciate the help from my colleagues and project collaborators in Samsung Electronics, Applied Materials, Lam Research and University of Michigan including Vladimir Volynets, John Yuan, Karl Leeser, and Mark Kushner.

\section{References}

[1] R. Doering and Y. Nishi, Ed., Handbook of Semiconductor Manufacturing Technology. Boca Raton, FL: CRC Press, 2008.

[2] S. Huang, V. Volynets, J. R. Hamilton, S. Lee, I. Song, S. Lu, J. Tennyson, M. J. Kushner, "Insights to scaling remote plasma sources sustained in NF3 mixtures," J. Vac. Sci. Technol. A, vol. 35, no. 3, 2017. 